\title{
Modeling the Performance of Cross-Flow Filtration Based on Particle
}

\section{Adhesion}

\author{
Chi Tien
}

and

\section{Bandaru V. Ramarao*}




\begin{abstract}
A mathematical model for predicting filtrate flux decay in cross flow is given. The formation of a cake in cross-flow filtration results in a decay of the filtrate flux. The growth of the cake is dependent upon the shear at the cake's surface and the filtrate flow normal to it. It is possible to predict the growth of the filter cake thickness in cross-flow based the concept that the cake surface growth is controlled by the adhesion of particles. This yields criteria for growth and also determines the filtrate flux and its decay. Unlike most previous studies, the parameters required for estimating the filtrate flux can be calculated directly based on operating conditions or they can be determined from independent measurements. Example calculation results are given and further improvement of the procedure discussed.
\end{abstract}

Key Words: Cake Filtration, Cross-Flow, Filtration Performance, Particle Adhesion, Filtration Model, Microfiltration 


\section{Introduction}

The development of membrane process for separating and removing particles and/or macromolecules from suspensions/solutions has reached the stage that its use has become a standard industrial practice nowadays. For cases where particle size is comparable to membrane pore size, the extent of particle penetration into membrane media can be expected to be insignificant except initially and cake formation and growth takes place over membrane surface. The cake thickness increases with time and the extent of growth depends upon the mode of filtration as well as operating variables.

The main features of cross-flow cake filtration may be summarized as follows:

1. There exists a threshold filtration velocity (critical flux) below which flux decline with time is absent $(1,2)$.

2. There is preferential deposition of smaller particles in cross-flow filtration of polydisperse suspensions $(3,4,5)$. In fact, the phenomenon of preferential deposition provides the principle in developing a particle size separation apparatus (5).

3. For constant pressure operation, filtration rate decreases and the rate of decrease diminishes with time. For many cases, there appears to be an attainment of steady state operation.

The decline of filtration rate in constant pressure filtration can be largely attributed to the growth of filter cakes at medium surfaces. However, if one assumes that all particles transported to membrane surface due to filtrate flow result in cake formation, the cake resistance would be so large and would give a filtration rate much less than the 3 
observed value (by orders of magnitude) This so-called flux paradox was first mentioned by Green and Belfort (6).

In formulating models of cross-flow filtration, it is important that the models formulated should be compatible with the above-mentioned features. Furthermore, as a practical matter, the models should provide sufficiently accurate filtration performance predictions without resorting to excessive computation.

For the past two decades and especially during more recent years, a large number of studies aimed at analyzing cross-flow cake filtration have appeared in the literature. Most of these investigations are concerned with specific systems and development of empirical models. As the parameters of the formulated models are not known a priori, their use in design calculations are limited.

A rigorous analysis of cake filtration may be made by considering the problem as one concerned with the motion of a large number of discrete particles together with the motion of their suspending fluid. Such a problem, in principle, may be solved by satisfying the Navier-Stokes equation at every point of the fluid and the equation of motion for each particle. This approach, of course, is too involved to be impractical. Not only is the number of particles too large to be handled individually, the fact that both the particle and fluid domains are not fixed but vary with time make the necessary computation practically impossible.

A simplified analysis of cross-flow filtration can be made by considering the physical domain of interest to be composed of two parts: A suspension phase which may be treated as a simple fluid and a cake region which provides resistance to filtrate flow. 4 
To resolve the flux paradox problems, particle transport away from the cake/suspension interface should be included in formalizing the cake growth expression. Using such an approach, Romero and Davis $(7,8)$ obtained numerical solutions of the two dimensional convective diffusion equation for both steady and unsteady state cases. The shear induced diffusion was assumed to be responsible for particle back transport. For cake formation, Romero and Davis assumed the cake region to be made up of two parts, a flowing part with solidosity (particle volume fraction) ranging from $\varepsilon_{\mathrm{s}_{\mathrm{o}}}$, the solid fraction of the feed suspension to $\left(\varepsilon_{\mathrm{s}}\right)_{\max }$, the so-called maximum packing density, and a stationary part with its solidosity being $\left(\varepsilon_{\mathrm{s}}\right)_{\max }$.

A similar but simpler analysis was later made by Song and Elimelech (9). ${ }^{*}$ The analysis was based on a steady-state one-dimensional convective diffusion equation assuming that the Brownian diffusion was responsible for particle back transport. Similar to the work of Romero and Davis (but using different terminology), the cake region

* There are a number of mistakes of the work of Song and Elimelech. In their Equations (3.9) - (3.11), particle concentration instead of excess particle concentration should be used. The lower integration limit of Equation (3.2.6) should be $\theta_{\mathrm{o}}$ corresponding to $\mathrm{c}_{\mathrm{o}}$ instead of "o". 
membrane is assumed to be composed of two parts; a polarized layer (same as the flowing cake part) and a cake layer (stationary) with assumed solidosities.

Analysis of cross-flow cake filtration based on steady state, one dimensional convective diffusion equation was also made by Bacchin et al (10). Similar to the study of Romero and Davis, the shear induced diffusion was used to account for particle back transport. A new feature of the work of Bacchin et al is the recognition that cake formation takes place if the particle concentration reaches a certain threshold value (or namely, the solidosity at the zero stress state, $\varepsilon_{\mathrm{s}}^{0}$, or the so-called gel point value) and the supposition that this threshold value can be determined for the osmotic pressure data, an interesting idea which remains to be validated.

In a more recent study, Kromkamp et al (11) presented results based on the numerical solution of two-dimensional convective diffusion equations using the LatticeBoltzmann method. Some of the assumptions used in the earlier work of Romero and Davis were relaxed. However, the assumption regarding particle back transport and cake structure suggested by Romero and Davis were also used in this work as well.

Before presenting a new and practical method for predicting cross-flow cake filtration performance, it is, perhaps proper to inquire: To what degree the previous studies mentioned above may be used for such purpose? It is beyond the scope of the present study to provide a thorough review of previous studies on this subject. Instead, a brief mentioning of a few salient points may be in order.

Large scale applications of membrane filtration such as water treatment inevitably require frequent back washing and periodic chemical cleaning of membrane modules 6 
suggesting unsteady state operation and invalidating the steady state assumption used in some analyses.

The use of the Brownian diffusion alone for particle back transport is contrary to the observation of preferential deposition of smaller particles. The limited shear induced diffusion data available, the significant differences among the available data and more importantly, that these data were obtained from measurements using particles of sizes of two or three order of magnitude greater than those found in membrane filtration makes the use of these results questionable, at least, in their accuracy. The assumption that cake solidosity may approach the maximum packing density value is contrary to all available experimental evidence.

The computation required for some of the analyses mentioned above may be excessive and not be practical for preliminary calculations. In view of what is mentioned above, the present study was undertaken. The purpose of the present study is to formulate a simple and rational method for predicting cross-flow cake filtration performance. The parameters and data required can be estimated based on operating conditions and/or determined for independent measurement. Studies of microfiltration including flux decay during cross flow filtration can be analyzed by using a model such as the adhesion concept based model described below. Details of the proposed method are given in the following sections. 


\section{Description of the Method of Prediction}

\subsection{Generalization of the Conventional Cake Filtration Theory.}

The classical conventional cake filtration theory based on the work of Ruth (12) with filtration carried out in the dead end mode may be written as (13)

$$
q=\frac{P}{\mu\left[s \rho V(1-\bar{m} s)^{-1}\left(\alpha_{a v}\right)_{\Delta p_{s}}+R_{m}\right]}
$$

where $q$ is the instantaneous filtration velocity, $\mathrm{P}$, the operating pressure, $\mathrm{V}$ the cumulative filtrate volume (and $q=\mathrm{dV} / \mathrm{dt}$ ), $\mathrm{R}_{\mathrm{m}}$, the medium resistance, $\rho$ and $\mu$ the filtrate density and viscosity, respectively. $\mathrm{s}$ is the particle mass fraction of the feed suspension and $\overline{\mathrm{m}}$ is the wet to dry cake mass ratio. $\left(\alpha_{\mathrm{av}}\right)_{\Delta \mathrm{p}_{\mathrm{c}}}$ is the average specific cake resistance of a filter cake subject to a pressure drop across the cake and is given as

$$
\left(\alpha_{\mathrm{av}}\right)_{\Delta \mathrm{p}_{\mathrm{c}}}=\frac{\Delta \mathrm{p}_{\mathrm{c}}}{\int_{\mathrm{o}}^{\Delta \mathrm{p}_{\mathrm{c}}}\left(\frac{1}{\alpha}\right) \mathrm{dp}_{\mathrm{s}}}
$$

and the pressure drop across filter cake is

$$
\Delta p_{c}=P-\mu q R_{m}
$$

where $\alpha$ is the specific resistance defined as $\alpha=\left(\mathrm{k} . \rho_{\mathrm{s}} \varepsilon_{\mathrm{s}}\right)^{-1}$ with $\mathrm{k}, \rho_{\mathrm{s}}$ and $\varepsilon_{\mathrm{s}}$ being the cake permeability, particle density and cake solidosity (particle volume fraction). For 8 
compressible cakes, $\mathrm{k}$ and $\varepsilon_{\mathrm{s}}$ are functions of the compressible stress. Equation (2.a) is based on the assumption that $\mathrm{p}_{\ell}+\mathrm{p}_{\mathrm{s}}=$ constant with $\mathrm{p}_{\mathrm{l}}$ and $\mathrm{p}_{\mathrm{s}}$ being the pore liquid pressure and cake phase compressive stress. Generally speaking, for constant pressure filtration $\left(\alpha_{\mathrm{av}}\right)_{\Delta \mathrm{p}_{\mathrm{c}}}, \mathrm{R}_{\mathrm{m}}$ and $\overline{\mathrm{m}}$ can be expected to be monotonically increasing functions of time but approach asymptotically to their respective constant values as the cake thickness increases.

For cross-flow filtration, Equation (1) may be modified to give (13)

$$
\begin{aligned}
q= & \frac{P}{\mu s \rho \alpha_{a v} \int_{o}^{t} \frac{\beta q d t}{1-s[(\bar{m}-1) \beta+1]}+\mu R_{m}} \\
& \cong \frac{P}{\frac{\mu s \rho \alpha_{a v}}{1-s} \int_{o}^{t} \beta q d t+\mu R_{m}} \text { for }(\bar{m}-1) \beta<<1
\end{aligned}
$$

which can be simplified to

$$
q \cong \frac{P}{\mu s \rho \alpha_{a v} \int_{o}^{t} \beta q d t+\mu R_{m}} \text { for } s<<1
$$

where $\beta$ is the fraction of particles transported to the medium surface which are deposited to form filter cakes. Details of the derivation of Equation (3.a) are shown in the appendix.

9 
Equation (1) can be seen to be a special case of Equation (3). For dead-end cake filtration, all particles associated with filtration flux result in cake formation or $\beta=1$ and $V=\int_{o}^{\ell} q . d t$. Equation (3) reduces to Equation (1) since under normal operating conditions, $1-\overline{\mathrm{ms}}$ can be regarded as constant.

It should also be pointed out that the integral in the denominator of Equation (3) may approach a constant value as $t$ increases. If the integral does reach a constant value, $q$ also becomes constant or filtration is carried out at a steady state.

\section{Estimation of $\boldsymbol{\beta}$ Based on Particle Adhesion.}

Before Equation (3) may be applied for predicting cross-flow cake filtration ratio, the value of $\beta$ must be known. An adhesion model was developed by Stamatakis and Tien (14) to account for particle adhesion in cross-flow cake filtration. Their model depicting the situation of a spherical particle being transported to and making contact with cake surface is shown in Fig. 1. Over a length scale comparable to particle size, the cake/suspension interface is not smooth and its roughness may be described in terms of the number of protrusions of various heights. When a particle makes contact with the interface against a protrusion of height $\mathrm{h}$, the condition that it remains deposited is given as

$F_{q} \sqrt{\left(d_{p} / 2\right)^{2}-\left[\left(d_{p} / 2\right)-h\right)^{2}}>F_{p}\left(\frac{d_{p}}{2}-h\right)$ 
Namely, the clockwise moment caused by the tangential force acting on the particle of diameter $d_{p}, F_{p}$ is not sufficient to counter balance the counter-clockwise movement caused by $F_{q}$, the force along the normal direction. Equation (4) is subject to the constraint of $h<d_{p} / 2$. On the other hand, deposition may be assumed if $h>d_{p} / 2$. Thus, there is a threshold value of $h, h^{*}$ below which deposition may not take place. From Equation (4), $\mathrm{h}^{*}$ is found to be

$\mathrm{h} *=\left[1-\frac{1}{\left[\left(\mathrm{~F}_{\mathrm{p}} / \mathrm{F}_{\mathrm{q}}\right)^{2}+1\right]^{1 / 2}}\right] \frac{\mathrm{d}_{\mathrm{p}}}{2}$

Since the height varies, a particle adhesion probability, $\gamma$, may be defined as

$\gamma=\mathrm{p}\left(\mathrm{h} \geq \mathrm{h}^{*}\right)$

where $\mathrm{p}\left(\mathrm{h} \geq \mathrm{h}^{*}\right)$ is the probability of a particle encountering a protrusion with a height equal to or greater than $h^{*}$. Similarly, $1-\mathrm{p}\left(\mathrm{h} \geq \mathrm{h}^{*}\right)$ is the probability for a particle encounter with protrusion of a height less than $\mathrm{h}^{*}$.

If one assumes that the height distribution is uniform with $h$ ranging from $h_{\min }$ $\mathrm{h}_{\min }=0$, to $\mathrm{h}_{\max }=\mathrm{d}_{\mathrm{p}} / 2$, from Equation (5), one has

$\gamma=\frac{1}{\left[\left(\mathrm{~F}_{\mathrm{p}} / \mathrm{F}_{\mathrm{q}}\right)^{2}+1\right]^{1 / 2}}$ 
In the absence of any particle back transport, $\gamma$ may be taken as $\beta$ [see Equation (3)]. To relate $\beta$ with the operating condition of filtration, consider the simple case where the forces experienced by a contacting particle, $F_{p}$ and $F_{q}$ are only hydrodynamic. $F_{q}$, according to Stokes' law may be expressed as

$F_{q}=3 \pi \mu d_{p} q$

Note that we have ignored the hydrodynamic correction factor for this force in the vicinity of a surface, for simplicity's sake. For $\mathrm{F}_{\mathrm{p}}$, according to O'Neil (15), one has

$\mathrm{F}_{\mathrm{p}}=\left.1.7009\left[3 \pi \mu \mathrm{d}_{\mathrm{p}}\right](\mathrm{u})\right|_{\mathrm{d}_{\mathrm{p}} / 2}$

where the quantity 1.7009 is the hydrodynamic retardation correction factor. $\left.u\right|_{d_{p} / 2}$ is the filtrate velocity at a point of $\mathrm{d}_{\mathrm{p}} / 2$ away from the cake/suspension interface. If the suspension flow may be assumed to be the flow between two parallel plates, $\left.u\right|_{\mathrm{d}_{\mathrm{p}} / 2}$ may be approximated as

$\left.u\right|_{d_{p} / 2}=u_{\max }\left(2 d_{p} / b\right)$ 
where $b$ is the distance between the two plates. $u_{\max }$ is the maximum velocity and may be taken to be $1.5 \overline{\mathrm{u}}$ where $\overline{\mathrm{u}}$ is the cross flow velocity.

Combining Equations (7), (8), (9) and (10), $\beta$ is found to be

$$
\beta=\gamma=\left\{\left[\frac{1.7009)\left(3 \pi \mu d_{p}\right)(1.5 \bar{u})\left(2 d_{p} / b\right)}{3 \pi \mu d_{p} q}\right]^{2}+1\right\}^{-1 / 2}
$$

2.3 Prediction of Cross-flow Filtration Performance." As given above, cross-flow cake filtration performance can be obtained from the solution of Equation (3) with $\beta$ given by Equation (11). The strong non-linearity of Equation (3) precludes a closed-form analytical solution but approximate numerical solutions can be obtained by using integral or differential methods. In the following we describe two variants: a simple integral method and a differential method.

* Prediction of cross-flow cake filtration performance based on the adhesion concept has been made by a number of investigators in the past, $(14,16,17)$ but not from the solution of Equation (3). 
We first rewrite Equation (3) by separating the integral term on the left hand side and collect the remaining terms to the right hand side. The resulting equation is (in order to simplify the notation, we have used $\alpha$ for $\alpha_{\mathrm{av}}$ ),

$$
\int_{0}^{t} \beta q d t=\frac{P}{\frac{\mu s \rho}{(1-s)}(\alpha q)}-\frac{R_{m}}{\frac{s \rho}{(1-s)} \alpha}
$$

\subsubsection{Integral Method.}

From Equation (3), physically speaking, $q$ is the dependent variable and the independent variable. However, since both $\beta$ and $(\alpha)_{\Delta p_{c}}$ are explicitly related to $q$ [see Equations (11), (2.a) and (2.b)], for numerical solution of Equation (3), it is more convenient to obtain results of $\mathrm{t}$ as a function of $q$. The integral on the left hand side of Equation (12) can be replaced by its approximation using the trapezoidal rule.

Considering a set of values of $q_{\mathrm{i}}, \mathrm{i}=1,2, \ldots$ with $q_{\mathrm{i}}$ being the value of $q$ at $\mathrm{t}=\mathrm{t}_{\mathrm{i}}$ and

$$
q_{i}=q_{i-1}+\Delta q
$$

$$
\begin{aligned}
\int_{o}^{t_{i}} \beta q d t=[ & \left.\beta_{o} q_{o}+\beta_{1} q_{1}\right] \frac{\Delta t_{1}}{2}+\left[\beta_{1} q_{1}+\beta_{2} q_{2}\right] \frac{\Delta t_{2}}{2} \\
& +\ldots . .\left[\beta_{i-1} q_{i-1}+\beta_{i} q_{i}\right] \frac{\Delta t_{i}}{2} \\
& =\sum_{k=1}^{i-1}\left[\beta_{k-1} q_{k-1}+\beta_{k} q_{k}\right] \frac{\Delta t_{k}}{2}+\left[\beta_{i-1} q_{i-1}+\beta_{i} q_{i}\right] \frac{\Delta t_{i}}{2}
\end{aligned}
$$


where $\beta_{\mathrm{k}}$ is the value of $\beta$ obtained from Equation (11) with $\mathrm{q}=\mathrm{q}_{\mathrm{k}}$ and

$\Delta \mathrm{t}_{\mathrm{k}}=\mathrm{t}_{\mathrm{k}}-\mathrm{t}_{\mathrm{k}-1}, \quad \mathrm{k}=1,2, \ldots \mathrm{i}$

Substituting Equation (14) into (12), after rearrangement, one has

$$
\left.\frac{P}{q_{i}}-\mu R_{m}=\frac{\mu s \rho}{1-s} \alpha_{i}\left[\sum_{k=1}^{i-1}\left\{\beta_{k-1} q_{k-1}+\beta_{k} q_{k}\right\}\right] \frac{\Delta t_{k}}{2}+\frac{\Delta t_{i}}{2}\left\{\beta_{i-1} q_{i-1}+\beta_{i} q_{i}\right\}\right]
$$

and $\alpha_{\mathrm{i}}$ is the value of $\alpha_{\mathrm{av}}$ calculated from Equation (2.a) with $\Delta p_{c}$ from Equation (2.b) with $q=q_{i}$.

From the above expression, one has

$$
t_{i}-t_{i-1}=\frac{2(1-s)}{\mu \rho \rho \alpha_{i}}\left[\frac{P / q_{i}-\mu R_{m}}{\beta_{i-1} q_{i-1}+\beta_{i} q_{i}}\right]-\sum_{k=1}^{i-1}\left[\frac{\beta_{k-1} q_{k-1}+\beta_{k} q_{k}}{\beta_{i-1} q_{i-1}+\beta_{i} q_{i}}\right] \Delta t_{k}
$$

The first step is an initialization step and can be calculated from the following equation.

$$
t_{1}=\frac{2(1-s)}{\mu \rho \rho \alpha_{1}}\left[\frac{P / q_{i}-\mu R_{m}}{\beta_{o} q_{o}+\beta_{i} q_{i}}\right]
$$

$$
\text { with } q_{1}=q_{o}+\Delta q \text {. }
$$


Equation (12) together with Equations (2.a), (2.b) and (3) constitute the system of equations describing the performance of cross-flow cake filtration. Numerical results of these equations may be obtained by specifying the value of $\Delta q$. Intuitively, one may expect that the accuracy of the numerical results improves with the decrease of $\Delta q$.

Note that Equations (18) and (17) are linear and explicit for the time steps, t. Another way to solve the above system of equations is to begin with a set of discrete time steps, $\left[0, \mathrm{t}_{1}, \ldots, \mathrm{t}_{\mathrm{i}}, \ldots \mathrm{t}_{\mathrm{N}}\right]$ and determine the corresponding liquid flux values, $\mathrm{q}_{\mathrm{i}},[\mathrm{i}=0, \ldots \mathrm{N}]$ from Equations (17) and (18). However, the equations for $\mathrm{q}_{\mathrm{i}}$ are no longer linear nor explicit (note that the deposition probabilities $\beta$ 's are functions of $q$ ) and non-linear implicit equation solvers have to be used.

\subsubsection{Differential Method.}

It is possible to convert Equation (12) into a first order ordinary differential equation by differentiation with respect to t to yield,

$$
\beta q=\frac{P(1-s)}{\mu s \rho} \frac{d}{d t}\left[\frac{1}{\alpha q}\right]-\frac{(1-s) R_{m}}{s \rho} \frac{d}{d t}\left[\frac{1}{\alpha}\right]
$$

Expanding the time derivatives (note that $\alpha$ is an implicit function of time through q), we obtain

$$
\frac{d q}{d t}=\frac{\beta q}{\left\{\frac{(1-s)}{s \rho} \frac{R_{m}}{\alpha^{2}} \frac{d \alpha}{d q}-\frac{P(1-s)}{\alpha^{2} q \mu s \rho} \frac{d \alpha}{d q}-\frac{P(1-s)}{q^{2} \alpha \mu s \rho}\right\}}
$$


This equation can be solved by quadrature after replacing the derivative $\mathrm{d} \alpha / \mathrm{dq}$ by a suitable finite difference approximation.

$\frac{d \alpha}{d q} \sim \frac{\alpha\left(q_{i}\right)-\alpha\left(q_{i-1}\right)}{\left(q_{i}-q_{i-1}\right)}$

Approximation 1:

If we assume that $\alpha$ and $\beta$ are constants, Equation (20) can be solved subject to the initial condition that the flux is $\mathrm{q}_{0}$. The resulting equation is

$q_{a 1}=\frac{1}{\left[\frac{1}{q_{0}^{2}}+\frac{2 \beta_{0} \alpha_{0} \mu s \rho}{P(1-s)} t\right]^{1 / 2}}$

where we have denoted $\mathrm{q}_{\mathrm{a} 1}$ as the approximation of $\mathrm{q}$ (denoted Appx 1 in the calculations).

Approximation 2:

We can also determine $q_{a}$ by assuming that the constants $\alpha$ and $\beta$ are evaluated at the current time [which can be done through Equations (20) and (21) if the liquid flux q is known]. The resulting equation is given as

$$
q_{a 2}=\frac{1}{\left[\frac{1}{q_{0}^{2}}+\frac{2 \beta \alpha \mu s \rho}{P(1-s)} t\right]^{1 / 2}}
$$


(denoted Appx 2 in the calculations).

\section{An Example}

As an example to illustrate the methods given above, we calculated the performance variables for cross-flow filtration of a sample calcium carbonate suspension. The conditions used are shown in Table 1.

The average specific cake resistance, $\alpha_{a v}$, is calculated according to

$$
\alpha_{a v}=\frac{\alpha^{0}\left(\Delta p_{c} / p_{s}\right)}{\int_{o}^{\Delta p_{c} / P a} \frac{d p_{s}^{*}}{\left(1+p_{s}^{*}\right)^{0.44}}}=\frac{\alpha^{0}\left[\left(p-q \mu R_{m}\right) / p_{A}\right]}{\left(P-q \mu R_{m}\right) / p_{A}} \frac{d p_{s}^{*}}{\left(1+p_{s}^{*}\right)^{0.44}}
$$

With $\alpha_{\mathrm{av}}$ calculated from the above expression and $\beta$ from Equation (11) numerical results of t vs. $q$ were calculated according to Equation (17) using three different values of $\Delta q\left(10^{-3}, 5 \times 10^{-4}, 2 \times 10^{-4}\right.$ and $\left.10^{-4}\right)$. Based on the numerical results, by interpolation, filtration performance expressed as $q$ vs. $\mathrm{t}$ was obtained. These results are shown in Tables 2-4 and Fig. 2 and 3. Table 2 shows the filtrate flux q for different times by the integral method (columns 2-4) and by the differential method (column 5). The calculations using the integral method converge rapidly as the step size $\Delta q$ is decreased. The step size of $10^{-4}$ may be taken for the converged solution although it is clear from these results that larger steps can yield reasonably accurate solutions for practical purposes. Column 5 shows calculations of the corresponding time values by the differential method namely, the solution of Equation (20) and Equation (21). The 
agreement between the calculations of $\mathrm{q}$ by both methods is excellent. Table 3 shows calculations of $\beta, \Delta \mathrm{P}, \alpha$ and time corresponding to different values of $\mathrm{q}$. The pressure drop and the average specific filtration resistance are explicit functions of $\mathrm{q}$ [cf. Equations (11) and (23)] and hence can be calculated easily if one considers q as the independent variable. For the differential method, the flux q must be determined first by solving the differential equation [Equation (20)]. The pressure drop across the cake layer increases with time as does the average specific filtration resistance. The latter is responsible for the flux decay with time. The adhesion probability, $\beta$ is very close to unity because the particle size and cross flow velocity are small enough that the normal force (to the membrane) $F_{q}$ dominates over the tangential force $F_{p}$. Calculations of this probability are shown in Table 4 for different values of cross flow velocity and particle diameter. When the cross flow velocity is increased, the adhesion probability decreases because the tangential force is much larger in this case [cf. Equation (11)]. There is also a substantial decrease with time because the liquid flux is reduced causing a reduction in the normal force which aids deposition. The effect of particle size is shown in Table 4 (b). The deposition probability reduces strongly with particle size again as a consequence of increased tangential force suppressing deposition.

We also present the results of the calculations of the liquid flux $q$ as a function of time in Fig 2 for particles of diameter 2, 4 and $10 \mu \mathrm{m}$ and for cross flow velocities of 0.001 and $0.1 \mathrm{~m} / \mathrm{s}$. The flux decay is much stronger for the low cross flow velocity since the cake layer build-up is fastest and consequently the flow resistance increases rapidly. When the cross flow velocity is increased to $0.1 \mathrm{~m} / \mathrm{s}$, the cake layer build-up is retarded 19 
due to the reduced adhesion probability and the liquid flux remains higher. A similar effect is found when the particle size is increased as shown by the curves for 4 and $10 \mu \mathrm{m}$ particles. Fig 3 shows the effectiveness of the simple analytical approximations given in Equations (22.a) and (22.b). The liquid flux calculations by the integral method for 0.001 $\mathrm{m} / \mathrm{s}$ and for two different particles sizes $(1$ and $10 \mu \mathrm{m})$ are shown as baseline curves. Approximation 1 uses the initial $\alpha$ and $\beta$ values whereas approximation 2 uses the 'current time' values. The flux is dominated by the medium resistance during the initial period whereas subsequent cake development controls the flux at later times. Therefore approximation 1 yields results which are better in the initial stage whereas approximation 2 yields results which are closer to the numerical solution throughout the filtration period. These solutions are only approximations however since they don't accurately model the change in the adhesion probability and the average specific cake resistance with time.

\section{Discussion}

4.1 Results of Illustrative Example. A few remarks about the numerical results obtained may be in order. It is clear that the results depend on the values of $\Delta q$ used. However, the differences among the results are rather insignificant especially between those using $\Delta q=2 \times 10^{-4}$ and $\Delta q=10^{-4}$. One may, therefore, conclude that sufficiently accurate performance prediction can be made using $\Delta q$ to be one order of magnitude less than $q_{o}$.

The results also confirm the expectation that $\beta$ decreases with the decrease of $q$ (or the increase of time). The pressure drop across the cake increases with time. However, even 
with the elapse of sufficiently long time, for example, $t=4700 \mathrm{~s}$, the pressure drop across the cake, $\Delta \mathrm{p}_{\mathrm{c}}$, only represents $80 \%$ of the operating pressure. In other words, the assumption commonly invoked, namely, the medium resistance is negligible may not be valid.

One should also point out that while the rate of decrease of $q$ diminishes with time, it does not necessarily imply the attainment of steady state operation. According to Equation (3), a steady state operation is established at $t \geq t_{c}$ if the integral of Equation (3) becomes constant for $t \geq t_{c}$. This, in turn, means that $\beta$ vanishes for $t \geq t_{c}$. On the other hand, the adhesion probability, according to Equation (11), is always finite unless $q$ vanishes. Therefore, $q$ is a monotonic decreasing function of time and does not approach a constant value. We can analyze the long time behavior of $\mathrm{dq} / \mathrm{dt}$ using Equation (20).

4.2 Possible Improvement of the Method of Calculation. A key factor in the analysis of cross-flow cake filtration is the resolution and explanation of the so-called flux paradox. The method presented in this work attempts to resolve the paradox by considering the adhesion probability of particles transported to the suspension/cake interface. However, adhesion may not be the only explanation. In the following, we discuss the possible improvement of the adhesion approach.

4.2.1 Incorporation of Particle Back Transport Effect. As stated previously, several investigators have incorporated the particle back transport effect (either the Brownian diffusion or the stress induced diffusion) in the analysis of cross-flow cake filtration 
$(7,8,9,10,11)$. Although the use of the Brownian diffusion assumption for particle back transport alone leads to predictions contrary to experimental observations, this does not rule out its being considered as one of the factors in resolving the flux paradox issue.

To include the effect of particle back transport, let $\mathrm{J}_{\mathrm{B}}$ be the net particle flux. $\mathrm{J}_{\mathrm{B}}$ may be written as

$$
J_{B}=k_{B} \Delta \varepsilon_{s}
$$

If one assumes that $\varepsilon_{\mathrm{s}}$ (particle volume fraction) is continuous across the suspension/cake interface, $\Delta \varepsilon_{\mathrm{s}}$ may be assumed to be

$$
\Delta \varepsilon_{\mathrm{s}}=\varepsilon_{\mathrm{s}}^{\mathrm{o}}-\varepsilon_{\mathrm{s}_{\mathrm{o}}}
$$

where $\varepsilon_{\mathrm{s}}^{0}$, the value of $\varepsilon_{\mathrm{s}}$ at the zero-stress state is the cake solidosity at the cake surface.

One may express the backward particle transport flux as a fraction of the particle flux associated with filtrate flow), $\Phi$, defined as

$$
\Phi=\frac{J_{B}}{q \frac{\varepsilon_{s}^{o}}{1-\varepsilon_{s}^{o}}}=k_{B}\left(\varepsilon_{s}^{o}-\varepsilon_{s_{o}}\right) \frac{1-\varepsilon_{s}^{o}}{q \varepsilon_{s}^{o}}
$$


The fraction of particles transported to the suspension/cake which are deposited to form filter cake, $\beta$ [which appears in Equation (3)] may then be expressed as

$$
\beta=(1-\Phi) \cdot \gamma
$$

For an estimation of $\Phi$, Equation (25) may be rewritten as

$$
\Phi=\left(\frac{k_{B} d_{w}}{D}\right)\left(\frac{D}{d_{w}}\right)\left(\frac{1-\varepsilon_{s}^{o}}{\varepsilon_{s}^{o}}\right) \frac{\varepsilon_{s}^{o}-\varepsilon_{s_{o}}}{q}
$$

where $d_{w}$ is the characteristic length of the membrane module (diameter or its equivalent) and $\mathrm{D}$, the relevant diffusion coefficient. D may be taken to be either the Brownian diffusion coefficient, $\mathrm{D}_{\mathrm{BM}}$, or the shear induced diffusion coefficient, $\mathrm{D}_{\mathrm{Sh}}$ depending upon the back transport mechanism assumed. The Brownian diffusion coefficient may be calculated from the following equation

$$
\mathrm{D}_{\mathrm{BM}}=\frac{\mathrm{kT}}{3 \pi \mu \mathrm{d}_{\mathrm{p}}}
$$

where $\mathrm{k}$ is the Boltzman constant $\left(1.3805 \times 10^{-23} \mathrm{~J} \mathrm{~K}^{-1}\right)$ and $\mathrm{J}$, the absolute temperature $\mathrm{K}$. For the shear induced diffusion coefficient, $\mathrm{D}_{\mathrm{Sh}}$, Leighton and Acrivos (18), based on these data, presented the following correlation 


$$
\frac{\mathrm{D}_{\mathrm{Sh}}}{\mathrm{a}_{\mathrm{p}}^{2} \omega}=0.5 \varepsilon_{\mathrm{s}}^{2}\left[1+0.09 \exp \left(7 \varepsilon_{\mathrm{s}}\right)\right]
$$

A different version of the above correlation given by the same authors is (19)

$$
\frac{\mathrm{D}_{\mathrm{Sh}}}{\mathrm{a}_{\mathrm{p}}^{2} \omega}=0.33 \varepsilon_{\mathrm{s}}^{2}\left[1+0.5 \exp \left(8.8 \varepsilon_{\mathrm{s}}\right)\right]
$$

where $\omega$ is the shear rate. The Brownian diffusion coefficient, at a given temperature is a monotonically decreasing function of particle size. For example, at $\mathrm{T}=293 \mathrm{~K}$ and in aqueous medium, $\mathrm{D}_{\mathrm{BM}}$ is found to be $2.1 \times 10^{-12}, 2.1 \times 10^{-13}, 8.4 \times 10^{-14}$ and $4.2 \times 10^{-14}$ $\mathrm{m}^{2} \mathrm{~s}^{-1}$ for particles of radii of $0.1,1,2.5$ and $5 \mu \mathrm{m}$.

The shear induced diffusion coefficient, according to either Equation (29.a) or (29.b) increases with the increase of the shear rate and the second power of particle radius. It also depends strongly on the particle volume fraction (solidosity). For the purpose of estimating the extent of back diffusion, because of the substantial difference between these two correlations, the experimental data used in developing them may be used directly. Referring to Fig. 4 of Ref. (15), for $\varepsilon_{\mathrm{s}}=0.2$, one has

$$
\left(\frac{\mathrm{D}_{\mathrm{Sh}}}{\mathrm{a}_{\mathrm{p}}^{2} \omega}\right) /(0.2)^{2} \cong 0.5
$$

or 


$$
\frac{D_{S h}}{a_{p}^{2} \omega}=(0.02)
$$

Corresponding to the conditions of the illustrative examples, for fully developed laminar flow between two parallel plates, $\omega$ is found to be

$$
\omega=\left(\frac{\partial \mathrm{u}}{\partial \mathrm{y}}\right)_{\text {surface }}=\left(\frac{6}{\mathrm{~b}}\right) \overline{\mathrm{u}}=1.2 \times 10^{4} \mathrm{~s}^{-1}
$$

The shear induced diffusion coefficient for particles of different sizes are shown in Table 5.

The Sherwood number present in Equation $(27),\left(\mathrm{k}_{\mathrm{B}} \mathrm{d}_{\mathrm{w}}\right) / \mathrm{D}$, for laminar flow in a closed conduit is known to be a function of conduit length among other variables. However, for a conduit of sufficient length (as compared with the characteristic length of the conduit), it approaches to a constant value. If the result of the classical Graetz solution is used, one has (20)

$$
\mathrm{N}_{\mathrm{Sh}}=\frac{\mathrm{k}_{\mathrm{B}} \cdot \mathrm{d}_{\mathrm{w}}}{\mathrm{D}}=3.656
$$

The importance of back transport in cross-flow cake filtration can be seen from the value of $\Phi$ defined by Equation (27). For the illustrative example, if the particle back transport is caused by the Brownian diffusion with the values of the various quantities given below

$$
\begin{aligned}
& \frac{\mathrm{k}_{\mathrm{B}} \cdot \mathrm{d}_{\mathrm{w}}}{\mathrm{D}}=3.656 \\
& \mathrm{D}=4.2 \times 10^{-14} \mathrm{~m}^{2} \mathrm{~s}^{-1} \\
& \mathrm{~b}=10^{-3} \mathrm{~m}
\end{aligned}
$$


$\varepsilon_{\mathrm{s}}^{\mathrm{o}}=0.2$

$\varepsilon_{\mathrm{s}_{\mathrm{o}}}=(0.001 / 2655) /[(0.001 / 2655)+(0.999 / 1000)]=3.77 \times 10^{-4}$

and assuming $q$ to be $(1 / 5)(q)_{\mathrm{o}}$, or $10^{-3} \mathrm{~m} \mathrm{~s}^{-1}$, from Equation (27), $\Phi$ is found to be

$$
\Phi=(3.658) \frac{4.2 \times 10^{-14}}{10^{-3}} \frac{0.8}{0.2} \frac{0.2-3.77 \times 10^{-4}}{10^{-3}}=1.228 \times 10^{-7}
$$

In other words, particle back transport due to the Brownian diffusion under the conditions of the illustrative example is insignificant. It also shows that unless the value of $(\mathrm{D} / q)$ is increased by several orders of magnitude; for example, particles of nano-size and very low filtration velocity, the effect due to the Brownian diffusion is negligible or even non-detectable.

Similarly, for the case of the shear induced diffusion being operative, with the conditions of the illustrative example, one has

$$
\Phi=3.656 \frac{6.0 \times 10^{-9}}{10^{-3}}\left(\frac{0.8}{0.2}\right) \frac{0.2-3.77 \times 10^{-4}}{10^{-3}}=1.755 \times 10^{-2}
$$

Again the extent of particle backward diffusion is insignificant although the $\Phi$ value of this case is orders of magnitude greater than the Brownian diffusion case. However, the effect of the shear induced diffusion may become substantial for large particles, for example, for particles of $d_{p} \geq 20 \mu \mathrm{m}$.

\subsubsection{Inclusion of the London van der Waals Force in the Determination of $\gamma$. For}

the calculation of $\gamma$ given before, only the hydrodynamic forces were considered. Among the various forces omitted, the London van der Waals force perhaps has the most 
potential significance. A brief discussion of its inclusion in the calculation of $\gamma$ and the uncertainties associated with it is now presented.

The contact between a particle in transport with cake surface may be viewed as that between two particles of equal size as shown in Fig. 4. The London van der Waals force, $\mathrm{F}_{\mathrm{vW}}$ in its simplest expression (the so-called unretarded case) is given as (21)

$$
\mathrm{F}_{\mathrm{vw}}=\frac{\mathrm{Ha}_{\mathrm{p}}}{12 \delta^{2}}
$$

where $\mathrm{H}$ is the Hamaker constant and $\delta$ is the separate distance between the two particles. $\mathrm{F}_{\mathrm{vw}}$ is attractive and acting along the direction of the line connecting the two particle centers.

The components of $F_{v w}$ along the tangential and normal directions shown in Fig. 4 are

$\left(\mathrm{F}_{\mathrm{vw}}\right)_{\mathrm{p}}=\left(\mathrm{F}_{\mathrm{vw}}\right) \sin \theta$

$\left(\mathrm{F}_{\mathrm{vw}}\right)_{\mathrm{q}}=\left(\mathrm{F}_{\mathrm{vw}}\right) \cos \theta$

with the inclusion of the London van der Waals force, from Equations (8), (9), (31), (32.a) and (32.b), $F_{p}$ and $F_{q}$ are 


$$
\mathrm{F}_{\mathrm{p}}=\left.1.7009\left[3 \pi \mu \mathrm{d}_{\mathrm{p}}\right](\mathrm{u})\right|_{\mathrm{d}_{\mathrm{p}} / 2}+\frac{\mathrm{hd}_{\mathrm{P}}}{24 \delta^{2}} \sin \theta
$$

$$
\mathrm{F}_{\mathrm{q}}=3 \pi \mu \mathrm{d}_{\mathrm{p}} \mathrm{q}_{\ell_{\mathrm{m}}}+\frac{\mathrm{Hd}_{\mathrm{p}}}{24 \delta^{2}} \cos \theta
$$

and the adhesion probability, $\gamma$, can be found from Equation (7) with $F_{p}$ and $F_{q}$ given by the above expressions.

Inclusion of the London van der Waals force in the calculation of $\gamma$ requires the values of the Hamaker constant, $\mathrm{H}$, the separation distance, $\delta$ and the contact position between the two particles, $\theta$, which can be expected to vary from $-\pi / 2$ to $\pi / 2$. The value of $H$ for aqueous dispersions is known to lie in the range $3 \times 10^{-21}-10^{19} \mathrm{~J}(21,22)$. Krupp $(23)$ indicated that in the absence of an intervening liquid, the minimum separation distance of two solids is about $4 \AA$. There does not exist any direct means for establishing the minimum separation distance which is of the present interest. Using particle detachment force measurements and assumed values of the Hamaker constant, $\delta$ was found to be approximately $4 \mathrm{~nm}(24)$.

This lack of precise information makes it questionable to include the London van der Waals force in the calculation of $\gamma$. However, some qualitative assessment of the effect of including the London van der Waals force can be made as follows. First, it is simple to show that $\left(\mathrm{F}_{\mathrm{vw}}\right)_{\mathrm{q}}$ is always attraction while $\left(\mathrm{F}_{\mathrm{vw}}\right)_{\mathrm{p}}$ may be either positive or 
negative depending on the angle $\theta$. Therefore, as a conservative estimate of particle's escaping from adhesion, the condition $\theta=0$ is assumed. In other words, the effect of including the London van der Waals force on $F_{p}$, is ignored, thus enhancing the likelihood of particle adhesion.

The relative contributions of the hydrodynamic force and the London van der Waals force to particle adhesion can be represented by the ratio

$$
\frac{\left(\mathrm{F}_{\mathrm{vw}}\right)_{\mathrm{q}}}{\left(\mathrm{F}_{\mathrm{vw}}\right)_{\mathrm{hy}}}=\frac{\left(\mathrm{Hd}_{\mathrm{p}}\right) /\left(24 \delta^{2}\right)}{32 \pi \mu \mathrm{d}_{\mathrm{p}} \mathrm{q}_{\ell_{\mathrm{m}}}}=\frac{\mathrm{H}}{72 \pi \mu \delta^{2} \mathrm{q}_{\ell_{\mathrm{m}}}}
$$

where $\left(\mathrm{F}_{\mathrm{q}}\right)_{\text {hy }}$ is given by Equation (8). For the condition of the illustrative example and assuming that $\mathrm{H}=10^{-21}, \delta=4 \times 10^{-9} \mathrm{~m}^{\text {and }} \mathrm{q}_{\ell_{\mathrm{m}}}=\left(\mathrm{q}_{\ell_{\mathrm{m}}}\right)_{\mathrm{o}}=5 \times 10^{-3} \mathrm{~m}$, their ratio is found to be 0.055 . Therefore, the inclusion of the London van der Waals force is unimportant. However, entirely different conclusions may be reached if $\mathrm{H}$ is assumed to have greater value. Similarly, as filtration proceeds, $\mathrm{q}_{\ell_{\mathrm{m}}}$ decreases with time, the importance of including the London van der Waals force may increase and may become more pronounced. On the other hand, the importance of incorporating the London van der Waals force is reduced if the actual separation (or minimum approach) is substantially greater than $4 \mathrm{~nm}$ and/or with the presence of the repulsive double layer force in cases with charged particles.

The uncertainties involved in the estimation of $F_{v w}$ make its inclusion in the calculation of $\gamma$ impractical. In light of this, the calculation of $\gamma$ as given in the illustrative example, at best, is a crude approximation. Consequently, the predicted cake filtration performance can only be considered as preliminary. 29 
It should be mentioned that in cases where $\left(F_{v w}\right)_{q}$ is dominant, and $F_{q}$ remains essentially constant, the adhesion probability becomes nearly independent of time. Assuming that the extent of particle back transport is insignificant, $\beta$ is essentially constant. The cross-flow cake filtration behavior then becomes indistinguishable from that of the dead-end cse, but with an equivalent specific cake resistance equal to $\beta\left(\alpha_{\mathrm{av}}\right)$.

As a further remark, because of the complexities involved in particle contact and the resulting calculation of the particle-particle interaction forces, accurate theoretical calculation or independent experimental determination of $\gamma$ (or $\beta$ ) is unlikely. A more practical way of solving the problem is to determine $\beta$ by fitting experimental data to Equation (3) with the expectation that $\beta$ so obtained can be related to the various systems and operating variables, leading to the eventual development of correlations of $\beta$.

\section{Conclusion}

Based on a previous work (14), a simple procedure of predicting cross-flow cake filtration is presented. The predictions are consistent with experimental observations that the filtration velocity diminishes with time. However, it also reveals that some of the common assumptions associated with cross-flow cake filtration such as negligible medium resistance and the attainment of steady state operation may be questionable.

The new method introduced also allows a direct examination of the effect of particle back transport, which has often been alluded to in cake filtration studies. The results show that at least for microfiltration, particle back transport by the Brownian diffusion is likely insignificant. The so-called shear induced diffusion could play a more significant role than the Brownian diffusion, but its effect, nevertheless, is likely modest 30 
and also difficult to assess because of the uncertain values of the shear induced diffusion coefficient.

The possibility of including the particle-particle interaction force was explored briefly while inclusion of such interaction forces may be necessary in certain cases, the lack of the required information for calculating the interaction forces makes their inclusion not practical.

\section{ACKNOWLEDGEMENT}

Partial support for the filtration research of BVR was provided by the National Science Foundation (USA) Grant No. CMMI-1100180 to SUNY College of Environmental Science and Forestry, Syracuse NY. We are also grateful to the member companies of the Empire State Paper Research Associates Inc., Syracuse NY for support. 


\section{REFERENCES}

1. J.A. Howell, Sub-critical flux operation of microfiltration, J. Membrane Sci., 107 (1995) 165-171.

2. R.W. Field, D. Wu, J.A. Howell, B.B. Gupta, Critical flux concept for microfiltration fouling, J. Membrane Sci., 100 (1995) 252-272.

3. E. Fisher, J. Raash, Model tests of particle deposition at the filter-medium in cross-flow filtration, $4^{\text {th }}$ World Filtration Congress proc. Part II, pp. 9.6-9.16 (1986).

4. J. Murkes, C.G. Carlsson, Cross Flow Filtration: Theory and Practice, John Wiley and Sons, New York (1988).

5. J. Meier, G.-M. Klein, V. Kottke, Crossflow filtration as a new method of wet classification of ultrafine particles, Sep. Pur. Tech., 26 (2002) 43-50.

6. G. Green, G. Belfort, Fouling of ultrafiltration membrane: lateral migration and particulate trajectory model. Desalination 35 (1980), 129-147.

7. C.A. Romero, R.H. Davis, Global model of crossflow microfiltration based on hydrodynamic particle diffusion, J. Membrane Sci., 39 (1988) 157-185.

8. C.A. Romero, R.H. Davis, Transient model of crossflow microfiltration, Chem. Eng. Sci., 45 (1990), 13-25.

9. L. Song, M. Elimelech, Theory of concentration polarization in crossflow filtration, J. Chem. Soc. Faraday Trans., 91 (1995) 3389-3398.

10. P. Bacchin, D. Si-Hassen, V. Starov, M.J. Clifton, P. Aimar, A unifying model for concentration polarization, gel-layer formation and particle deposition in cross-flow membrane filtration of colloidal suspensions, Chem. Eng. Sci., 57 (2002) 77-91. 32 
11. J. Kromkamp, A. Bastiaanse, J. Swarts, G. Brans, R.G.M. van der Sman, R. M. Boom, A suspension flow model for hydrodynamic and concentration polarization in crossflow microfiltration, J. Mem. Sci., 253 (2005) 67-79.

12. B.F. Ruth, Studies in filtration III. Dirivation of general filtration equations, Ind. Eng. Chem. 27 (1935) 708-723.

13. C. Tien, Introduction to Cake Filtration: Analysis, Experiments and Application, Elsevier, 2007.

14. K. Stamatakis, C. Tien, A simple model of cross-flow filtration based on particle adhesion, AIChE J., 39 (1993) 1292-1302.

15. M.E. O’Neill, A sphere in contact with a plane while in a slow shear flow, Chem. Eng. Sci., 23 (1968) 1293-1298.

16. W.R. Bowan, H.S. Yousef, J.I. Calvo, Dynamic crossflow ultrafiltration of colloids: A deposition probability cake filtration approach, Sep. proc. Tech., 24 (2001) 297-308.

17. S. Chellam, M.R. Wiesner, Evaluation of crossflow filtration models based on shear-induced diffusion and particle adhesion: Complications induced by feed suspension polydispersity, J. Mem. Sci., 138 (1998) 83-97.

18. D.T. Leighton, A. Acrivos, Measurements of shear induced self diffusion coefficient in concentrated suspension of spheres, J. Fluid Mech, 177 (1987) 109-131.

19. D.T. Leighton, A. Acrivos, Viscous resuspension, Chem. Eng. Sci., 41 (1986), 1397-1384.

20. 
21. J.G. Knudsen, D, Kutz, Fluid Dynamics and Heat Transfer, p. 372, McGraw-Hill (1958).

22. D.J. Shaw, Introduction to Colloid and Surface Chemistry, $2^{\text {nd }}$ Ed., Butterworths, London-Boston, 1978.

23. M. Elimelech, J. Gregory, X. Jin, R.A. Williams, Particle Deposition and Aggregation: Measurements, Modeling and Simulation, Butterworths-Heinemann, 1995.

24. H. Krupp, Particle adhesion theory and experiments, Adv. in Colloid Interface Sci., 1 (1967) 112-239.

25. R. Vaidyanathan, Hydrosol Filtration in Granular Beds, M.S. Thesis, Syracuse Univ. (1986).

26. A. Hasan, L. R. Yasarla, B. V. Ramarao, T. E. Amidon. 'Separation of lignocellulosic components using ceramic microfilters.' J. Wood Chem. Technol., 31, 4, (2011) 357-365. 


\section{LIST OF TABLES AND FIGURES}

Table 1. Characteristics of filtering suspension.

Table 2. Numerical Results of $q$ vs. Time for different $\Delta \mathrm{q}$. [Time for given $q$ (min)] Results from Integral Method (Columns 2-5) and Differential Method (Column 6)

Table 3. Numerical Results of $\beta, \Delta P_{c}, \alpha_{a v}, q$, vs. time, $\Delta q=10^{-4} \mathrm{~ms}^{-1}$.

Table 4. Variation of the adhesion probability factor $\beta$, with cross flow velocity (a) and particle diameter (b).

Table 5. $\quad$ Estimated shear diffusivity for particles of different sizes.

Fig 1. Deposition according to the adhesion hypothesis.

Fig 2. Numerical Results of $q$ vs. Time for different particle diameters and cross flow velocities. [Top 3 curves are at cross flow velocity of $0.1 \mathrm{~m} / \mathrm{s}, \mathrm{d}_{\mathrm{p}}$ as labeled. Bottom curve is at cross flow velocity of $0.001 \mathrm{~m} / \mathrm{s}$ and $d_{p} 2 \mu \mathrm{m}$.]

Fig 3. Flux calculations using numerical method (Integral) and different approximations.

Fig 4. Forces acting between two particles. 
Table 1. Characteristics of filtering suspension.

$\begin{array}{ll}\text { Particle diameter, } \mathrm{d}_{\mathrm{p}} & 2 \times 10^{-6} \mathrm{~m} \\ \text { Particle mass fraction, } \mathrm{s} & 0.001 \\ \text { Operating Pressure, } \mathrm{P} & 30 \mathrm{kPa} \\ \text { Cross-flow velocity } & 2 \mathrm{~ms}^{-1} \\ \text { Medium Resistance, } \mathrm{R}_{\mathrm{m}} & 6 \times 10^{9} \mathrm{~m} \\ \text { Constitutive Relationship } & \alpha_{0}=3.85 \times 10^{10} \mathrm{mkg}^{-1} \\ \alpha=\alpha^{o}\left[1+\frac{p_{s}}{p_{A}}\right]^{n} & \mathrm{n}=0.44 \\ & \mathrm{p}_{\mathrm{A}}=4.4 \mathrm{kPa}^{n} \\ \text { Fluid Viscosity } & 10^{-3} \mathrm{~Pa} . \mathrm{s}^{-3} \\ \text { Fluid Density } & 1000 \mathrm{kgm}^{-3} \\ \text { Channel height, } \mathrm{b}, \quad \text { (for calculating } \beta \text { ) } & 10^{-3} \mathrm{~m}^{2}\end{array}$


Table 2. Numerical Results of $q$ vs. Time for different $\Delta \mathbf{q}$. [Time for given $q(\mathrm{~min})$ ] Results from Integral Method (Columns 2-5) and Differential Method (Column 6)

q

Time (min)

INTEGRAL SOLUTION

DIFFERENTIAL

SOLUTION

$$
\Delta q=10^{-4}
$$$$
\Delta \mathrm{q}=2 \times 10^{-4}
$$$$
\Delta q=5 \times 10^{-4}
$$$$
\Delta q=10^{-3}
$$

0.0005

0

0.0048

0.020703

0

0.020702

0

0

0

0.0046

0.04212

0.042116

0.0045

0.053283

0.0044

0.064843

0.064835

0.089421

0.0042

0.089435

0.116476

0.116317

0.115771

0.180951

0.053238

0.0208

0.0425

0.0538

0.0655

0.0903

0.0040

0.116499

0.181001

0.199969

0.266241

0.266143

0.199501

0.0032

0.0030

0.320472

0.320336

0.319398

0.316229

0.464833

0.0026

0.4651

0.511745

0.0025 


$\begin{array}{llllll}0.0022 & 0.689760 & 0.689210 & & 0.6962 \\ 0.0020 & 0.853523 & 0.852703 & 0.847128 & 0.829156 & 0.8615 \\ 0.0016 & 1.377982 & 1.37594 & & 1.3912 \\ 0.0015 & 1.577272 & & 1.556972 & 1.5925 \\ 0.0012 & 2.498041 & 2.491573 & & 3.292952 & 3.6550 \\ 0.0010 & 3.617563 & 3.604225 & 3.519753 & 10.2133 \\ 0.0006 & 10.08666 & 9.987714 & & & 14.7143 \\ 0.0005 & 14.51031 & & & & \\ 0.0002 & 88.95005 & 82.92091 & & & 363.330\end{array}$


Table 3. Numerical Results of $\beta, \Delta P_{c}, \alpha_{a v}, q$, vs. time, $\Delta q=10^{-4} \mathrm{~ms}^{-1}$

$\begin{array}{lllll}q \mathrm{~ms}^{-1} & \beta & \Delta \mathrm{P}_{\mathrm{c}}(\mathrm{Pa}) & \alpha(\mathrm{m} / \mathrm{kg}) & \text { Time }(\mathrm{min}) \\ 0.005 & 0.999998 & - & - & 0 \\ 0.0048 & 0.999998 & 1,200 & 4.07 \times 10^{10} & 0.02070 \\ 0.0045 & 0.999997 & 3,000 & 4.35 \times 10^{10} & 0.05328 \\ 0.0040 & 0.999997 & 6,000 & 4.75 \times 10^{10} & 0.11650 \\ 0.0035 & 0.999996 & 9,000 & 5.09 \times 10^{10} & 0.19997 \\ 0.0030 & 0.999994 & 12,000 & 5.40 \times 10^{10} & 0.32047 \\ 0.0025 & 0.999992 & 15,000 & 5.69 \times 10^{10} & 0.51174 \\ 0.002 & 0.999987 & 18,000 & 5.93 \times 10^{10} & 0.85352 \\ 0.0015 & 0.999977 & 21,000 & 6.16 \times 10^{10} & 1.57727 \\ 0.001 & 0.999948 & 24,000 & 6.39 \times 10^{10} & 3.61756 \\ 0.0005 & 0.999792 & 27,000 & 6.60 \times 10^{10} & 14.5103\end{array}$


Table 4. Variation of the adhesion probability factor $\beta$, with cross flow velocity (a) and particle diameter (b).

(a)

$\mathrm{q}_{1 \mathrm{~m}}, \mathrm{~m} / \mathrm{s}$

$\beta$

Channel Re

1

10

100

$\mathrm{U} \mathrm{ms}^{-1}$

0.001

0.010

0.100

0.0050

0.999997917

0.999791757

0.979798

0.0048

0.999997740

0.999774047

0.978136

0.0046

0.999997539

0.99975398

0.976262

0.0045

0.999997428

0.999742928

0.975234

0.0044

0.999997310

0.999731115

0.974140

0.0042

0.999997048

0.999704909

0.971724

0.0040

0.999996745

0.999674677

0.968959

0.0035

0.999995749

0.999575151

0.960020

0.0030

0.999994214

0.999421867

0.946719

0.0025

0.999991668

0.999167806

0.925828

0.0020

0.999986981

0.998700609

0.890734

0.0015

0.999976855

0.997693465

0.826783

0.0010

0.999947927

0.994832617

0.699875

0.0005

0.999791757

0.979798229

0.439962 
Table 5. Estimated shear diffusivity for particles of different sizes.

$\begin{array}{ll}\mathrm{a}_{\mathrm{p}}, \mathrm{m} & \mathrm{D}_{\mathrm{Sh}}, \mathrm{m}^{2} \mathrm{~s}^{-1} \\ 10^{-7} & 4 \times 10^{-12} \\ 10^{-6} & 2.4 \times 10^{-10} \\ 2.5 \times 10^{-6} & 1.5 \times 10^{-9} \\ 5 \times 10^{-6} & 6.0 \times 10^{-9}\end{array}$

(b)

q $\quad \beta$

$0.02 \quad 0.05$

$\left(\mathrm{d}_{\mathrm{p}} \mathrm{q} / \mathrm{v}\right)$

$d_{p} \mu m$

2

4

10

0.0050

0.979798

0.925828

0.699875 


\begin{tabular}{llll}
0.0048 & 0.978136 & 0.920254 & 0.685165 \\
0.0046 & 0.976262 & 0.914057 & 0.669566 \\
0.0045 & 0.975234 & 0.910697 & 0.661417 \\
0.0044 & 0.974140 & 0.907146 & 0.653028 \\
0.0042 & 0.971724 & 0.899414 & 0.635499 \\
0.0040 & 0.968959 & 0.890734 & 0.616930 \\
0.0035 & 0.960020 & 0.863838 & 0.565631 \\
0.0030 & 0.946719 & 0.826783 & 0.506813 \\
0.0025 & 0.925828 & 0.774615 & 0.439962 \\
0.0020 & 0.890734 & 0.699875 & 0.364914 \\
0.0015 & 0.826783 & 0.592179 & 0.282024 \\
0.0010 & 0.699875 & 0.439962 & 0.192313 \\
0.0005 & 0.439962 & 0.237929 & 0.097518 \\
0.0001 & 0.097518 & 0.048934 & \\
\hline
\end{tabular}




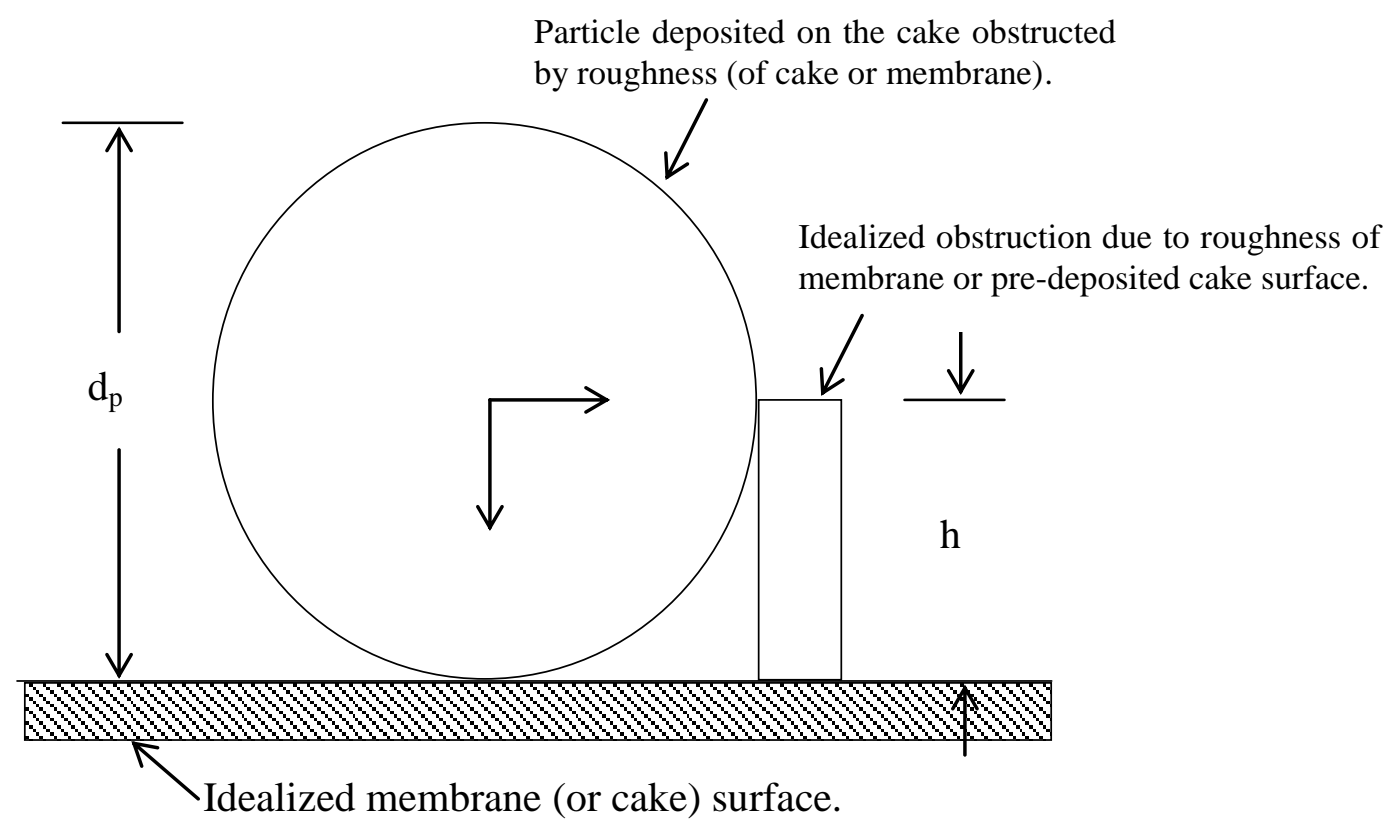

Fig 1. Deposition according to the adhesion hypothesis. 


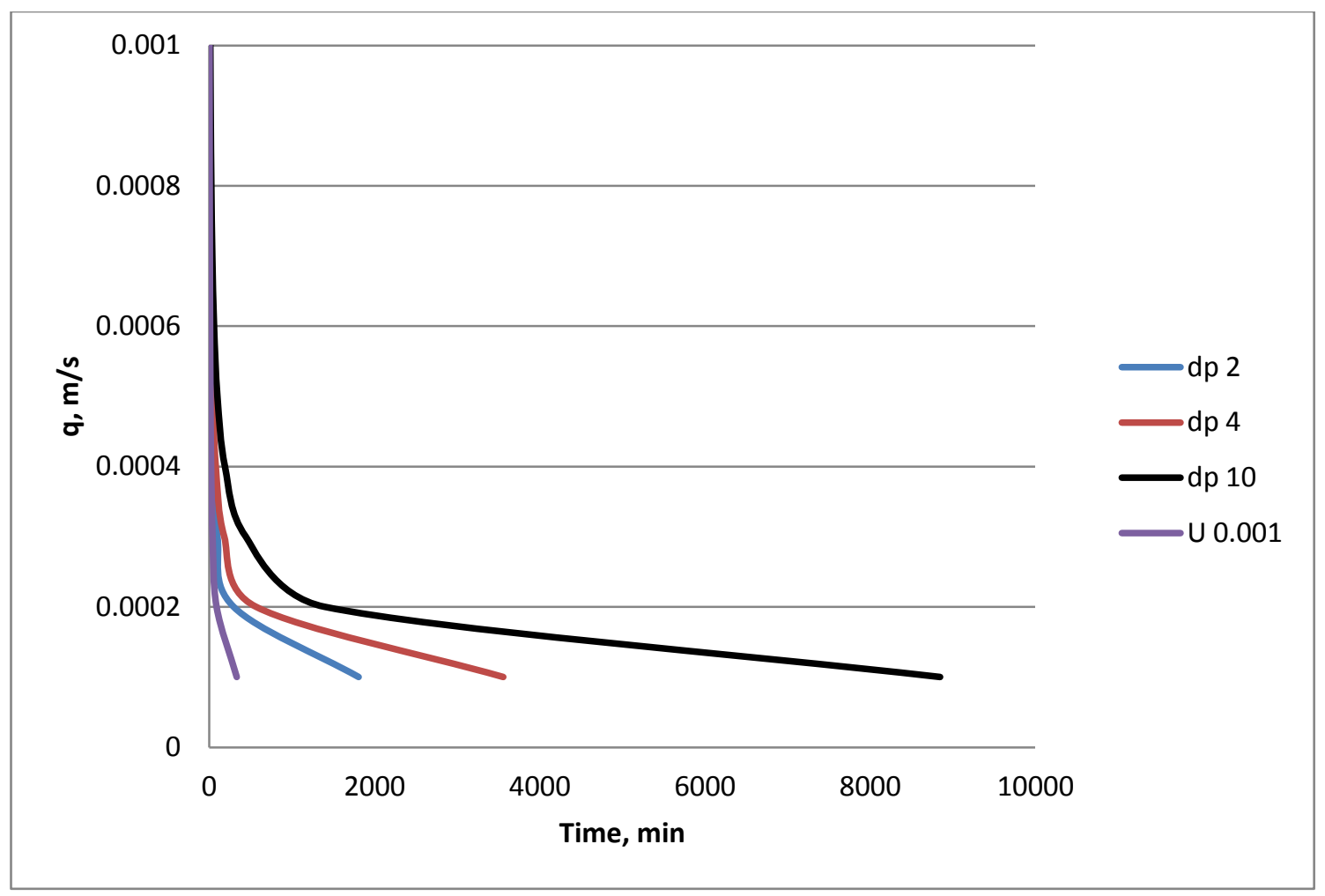

Fig 2. Numerical Results of $q$ vs. Time for different particle diameters and cross flow velocities. [Top 3 curves are at cross flow velocity of $0.1 \mathrm{~m} / \mathrm{s}, \mathrm{d}_{\mathrm{p}}$ as labeled. Bottom curve is at cross flow velocity of $0.001 \mathrm{~m} / \mathrm{s}$ and $\mathrm{d}_{\mathrm{p}} 2 \mu \mathrm{m}$.] 


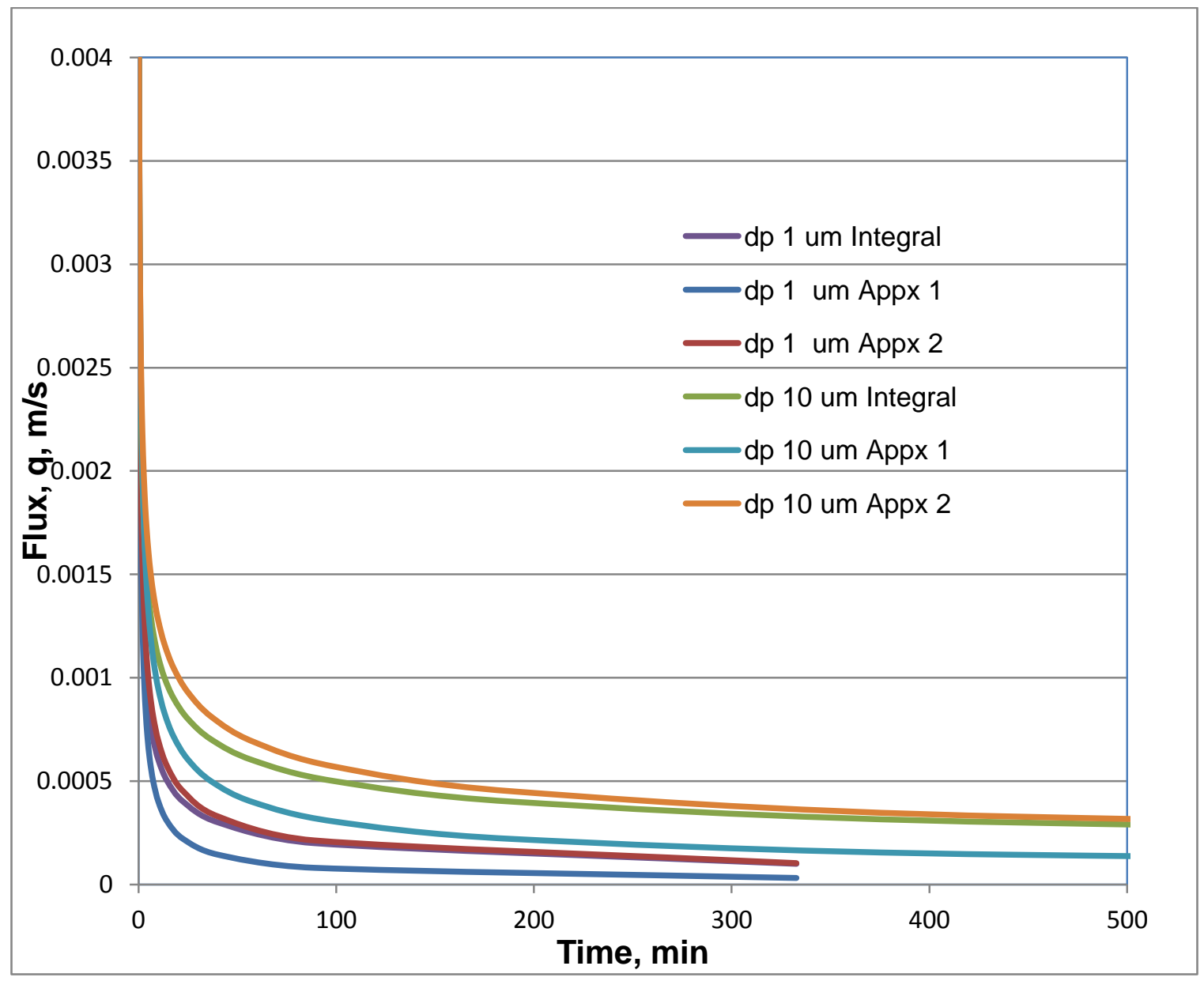

Fig 3. Flux calculations using numerical method (Integral) and different approximations. 


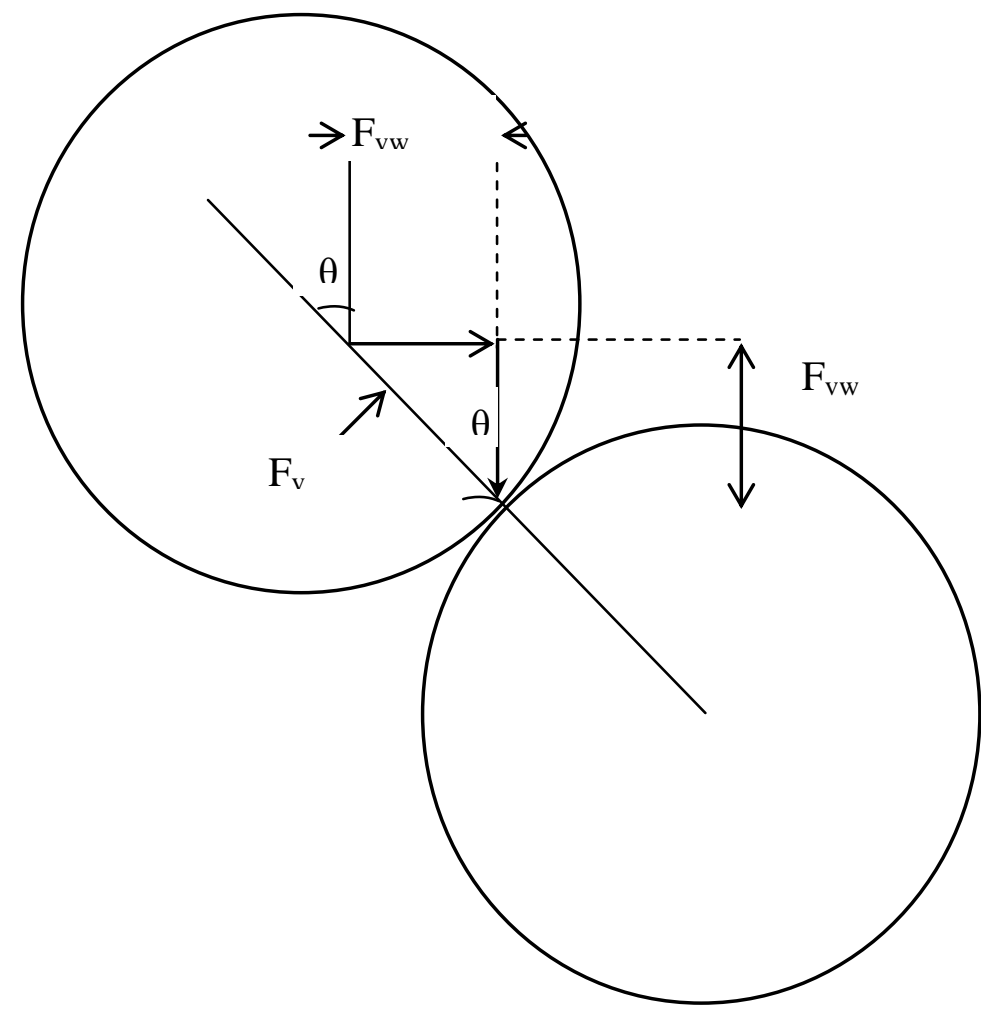

Fig 4. Forces acting between two particles. 